\title{
Comment on Lun et al. entitled "The prognostic value of elevated ezrin in patients with osteosarcoma"
}

\author{
Zhi Li • Youjun Li
}

Received: 5 December 2013 / Accepted: 19 December 2013 / Published online: 5 January 2014

(C) International Society of Oncology and BioMarkers (ISOBM) 2014

\section{Dear Editor,}

We read with great interest the recent article entitled "The prognostic value of elevated ezrin in patients with osteosarcoma" published online in September 2013 issue of Tumor Biology by Lun et al. [1]. The investigators conducted a meta-analysis to assess the prognostic value of elevated ezrin in patients with osteosarcoma. It is a very valuable study. Nevertheless, there are some comments we would like to raise related to this article.

To start with, only two electronic databases (PubMed and Embase databases) were systematically searched by the investigators. Small number of required papers would be an important limitation of the meta-analysis. We suggest at least three electronic databases should be systematically searched, such as OVID, the Cochrane Central Register, and so on. Search strategy report plays an important role in the meta-analysis. However, the investigators never mentioned a complete search strategy. In our opinion, they should provide us the flow diagram of search strategy and the manual search protocol.

Furthermore, publication language was limited to English in the meta-analysis. Therefore, there might exist a potential language bias in their meta-analysis. We suggest that no language restriction for the included studies to reduce the bias.

In addition, the investigators did not provide us any forest plots for meta-analysis and funnels for Begg's and Egger's tests. To strengthen the credibility of the meta-analysis, the investigators should provide us all these plots.

Eventually, the investigators did not provide us the basic characteristic of the included studies. To make us read this meta-analysis well, the investigators should provide us all these characteristic, such as age, sex, tumor size, chemotherapeutic regiments, histological type, and so on.

Moreover, more researches with large sample size are needed to further clarify the prognostic significance of ezrin expression in patients with osteosarcoma.

\section{Reference}

1. Lun DX, Hu YC, Xu ZW, Xu LN, Wang BW. The prognostic value of elevated ezrin in patients with osteosarcoma. Tumor Biol. 2013. doi: 10.1007/s13277-013-1168-2.

\footnotetext{
Z. Li • Y. Li $(\bowtie)$

Department of Human Anatomy and Histoembryology, Norman Bethune University of Medical Science, Changchun 130000, Jilin Province, People's Republic of China e-mail: youjun863@126.com

Z. Li

Central Hospital attached to Shenyang Medical College,

Shenyang 110024, Liaoning Province, People's Republic of China
} 